\title{
A blast without power - cell death induced by the tuberculosis-necrotizing toxin fails to elicit adequate immune responses
}

\author{
C Maueröder ${ }^{1}$, RA Chaurio ${ }^{1}$, T Dumych ${ }^{2,3}$, M Podolska $^{1}$, MD Lootsik ${ }^{2,3}$, S Culemann ${ }^{1}$, RP Friedrich $^{4}, \mathrm{R}$ Bilyy ${ }^{1,2}$, C Alexiou ${ }^{4}$, G Schett ${ }^{1}$, \\ C Berens ${ }^{5}$, M Herrmann $^{*, 1}$ and LE Munoz ${ }^{1}$
}

In this study, we deploy a doxycycline-dependent suicide switch integrated in a tumor challenge model. With this experimental setup, we characterized the immunological consequences of cells dying by four distinct cell death stimuli in vivo. We observed that apoptotic cell death induced by expression of the truncated form of BH3 interacting-domain death agonist (tBid) and a constitutively active form of caspase 3 (revC3), respectively, showed higher immunogenicity than cell death induced by expression of the tuberculosis-necrotizing toxin (TNT). Our data indicate that the early release of ATP induces the silent clearance of dying cells, whereas the simultaneous presence of 'find me' signals and danger-associated molecular patterns (DAMPs) promotes inflammatory reactions and increased immunogenicity. This proposed model is supported by findings showing that the production and release of high concentrations of IL-27 by bone-marrow-derived macrophages (BMDM) is limited to BMDM exposed to those forms of death that simultaneously released ATP and the DAMPs heat-shock protein 90 (HSP90) and high-mobility group box-1 protein (HMGB1). These results demonstrate that the tissue microenvironment generated by dying cells may determine the subsequent immune response.

Cell Death and Differentiation (2016) 23, 1016-1025; doi:10.1038/cdd.2016.4; published online 4 March 2016

Cell death and the clearance of dying cells have important roles in physiological and pathological processes. ${ }^{1}$ For instance, the silent elimination of excessive cells by apoptosis and their non-inflammatory clearance ${ }^{2}$ is necessary for tissue homeostasis ${ }^{3}$ and also has a fundamental role in development. ${ }^{4}$ In contrast, a deficiency in the clearance of apoptotic cells and the progression of the latter into secondary necrosis contributes to the etiopathogenesis of systemic lupus erythematosus. ${ }^{5}$ In cancer, an increased rate of apoptotic tumor cells is associated with poor prognosis. ${ }^{6}$

In this study, we aimed to investigate the immunological consequences of primary necrosis. Therefore, we used an established doxycycline-dependent suicide switch $^{7}$ integrated in a tumor challenge model as described previously. ${ }^{8,9}$ This system ensured that cell death, from the very beginning, occured in vivo.

The induction of necrosis by methods like heating or repeated freeze-thaw cycles strongly differs from necrosis induced by accidental damage. It is unclear whether shortlived signals released from dying or dead cells are preserved and how protein denaturation affects downstream responses.
We therefore relied on the induction of primary necrosis by expression of the tuberculosis-necrotizing toxin (TNT). TNT is derived from Mycobacterium tuberculosis (MTB) and induces direct progression of cells into primary necrosis. ${ }^{10}$ Cell death is induced by glycohydrolysis of $\mathrm{NAD}^{+}$without the activation of apoptotic or necroptotic pathways. ${ }^{10,11}$

To better assess the immunological consequences of necrosis induced by $\mathrm{NAD}^{+}$depletion, we included three apoptotic stimuli in this study. (1) Apoptosis induced by expression of the truncated form of $\mathrm{BH} 3$ interacting-domain death agonist (tBid). Bid is a pro-apoptotic protein, which is activated upon cleavage by Caspase-8. tBid interacts with $\mathrm{Bcl}-$ 2 -associated $X$ protein (Bax) thereby facilitating the integration of the latter in the mitochondrial outer membrane, which ultimately leads to mitochondrial outer membrane permeabilization. (2) Apoptosis induced by expression of a constitutively active form of Caspase-3 (revC3). Caspase-3 is a promiscuous executioner caspase involved in the downstream processes of apoptosis. ${ }^{12}$ It consists of a long subunit (LS) preceding a small subunit (SS). During apoptosis, initiator

\footnotetext{
${ }^{1}$ Department of Internal Medicine 3 - Rheumatology and Immunology, Friedrich-Alexander University of Erlangen-Nuremberg, Erlangen, Germany; ${ }^{2}$ Danylo Halytsky Lviv National Medical University, Lviv, Ukraine; ${ }^{3}$ Institute of Cell Biology, National Academy of Sciences of Ukraine, Lviv, Ukraine; ${ }^{4}$ ENT Clinic, Section of Experimental Oncology and Nanomedicine (SEON), Else Kröner-Fresenius-Stiftung Professorship, University Hospital Erlangen, Erlangen, Germany and ${ }^{5}$ Institute of Molecular Pathogenesis, Friedrich-Loeffler-Institut, Federal Research Institute for Animal Health, Jena, Germany

*Corresponding author: M Herrmann, Department of Internal Medicine 3 - Rheumatology and Immunology, Friedrich-Alexander University of Erlangen-Nuremberg, Krankenhausstrasse 12, Erlangen 91054, Germany. Tel: +49 913185 36990; Fax: +49 913185 35776; E-mail: martin.herrmann@uk-erlangen.de

Abbreviations: A1, Bcl-22-related protein A1; ANOVA, analysis of variance; ATP, adenosine triphosphate; AxV, recombinant chicken annexin A5; Bax, Bcl-2-associated X protein; Bid, BH3 interacting-domain death agonist; BMDM, bone-marrow-derived macrophages; DAMP, danger-associated molecular pattern; DIC, differential interference contrast; FITC, fluorescein isothiocyanate; HMGB1, high-mobility group box-1; HSP, heat-shock protein; IFN, interferon; IL, interleukin; LS, long subunit; LPS, lipopolysaccharide; Mcl-1, myeloid cell leukemia 1; MHC, major histocompatibility complex; MTB, Mycobacterium tuberculosis; NAD+, nicotinamide adenine dinucleotide; $\mathrm{PI}$, propidium iodide; revC3, reverse Caspase 3; ROS, reactive oxygen species; SS, small subunit; tBid, truncated BH3 interacting-domain death agonist; TNF, tumor necrosis factor; TNT, tuberculosis-necrotizing toxin; UVB, ultraviolet B

Received 30.10.15; revised 25.12.15; accepted 29.12.15; Edited by G Kroemer; published online 04.3.16
} 
caspases cleave Caspase-3, allowing LS and SS to reassemble and form active heterodimers. By reversing the arrangement of LS and SS, a constitutively active form of Caspase-3 (revC3) is created, mimicking the molecular structure of the cleaved variant. ${ }^{13}$ (3) Apoptosis induced by irradiation with UVB, which is characterized by death receptor activation and the early production of reactive oxygen species (ROS). ${ }^{14}$

In addition to the general interest of how different forms of cell death affect the immune system, our findings may help to further understand MTB infection pathogenesis. Cell death induced by this pathogen has long been described as atypical in a negative manner, lacking features of apoptosis, pyroptosis and necroptosis. ${ }^{15}$ Furthermore, this study provides an alternative perspective on whether induction of primary necrosis is a safe bet for the induction of tumor immunity.

\section{Results}

Conditional expression of the suicide proteins was induced by adding doxycycline, and the progression of cell death was monitored as reported. ${ }^{16}$ The cells were analyzed with regard to mitochondrial membrane potential, condition of the nucleus, phosphatidylserine exposure and plasma membrane integrity (Figure 1). Conditional expression of tBid induced rapid cell death characterized by early mitochondrial dysfunction and progression into apoptotic and secondary necrotic phenotypes (Figure 1b). The addition of doxycycline to the cell line B644-revC3 also induced apoptosis, however, with markedly slower kinetics and less involvement of the mitochondria (Figure 1c). In contrast, TNT-induced cell death was devoid of apoptotic cells and the cells directly progressed in primary necrosis (Figure 1d). To visualize the changes accompanying cell death, we used live cell imaging. Conditional expression of tBid induced the early loss of mitochondrial membrane potential before any morphological features of apoptosis became visible. Subsequently, the cells rounded up and detached, first exposing phosphatidylserine followed by the loss of plasma membrane integrity as indicated by propidium iodide staining (Supplementary Figure S1, Supplementary Movie 1). In contrast, the addition of doxycycline to the cell line B644-revC3 induced early rounding, detachment and blebbing, but the cells maintained their mitochondrial membrane potential for a longer period of time. At later stages of cell death, the cells exposed phosphatidylserine and then progressed into secondary necrosis (Supplementary Figure S2, Supplementary Movie 2). The expression of TNT induced early rounding and the decrease of cellular adhesion, followed by the concomitant loss of mitochondrial potential and plasma membrane integrity (Supplementary Figure S3, Supplementary Movie 3).

We first investigated whether different forms of cell death are able to overcome the allogeneic rejection of a secondary challenge with viable tumor cells. Implantation of viable B644 melanoma cells into Balb/c mice resulted in tumor growth and subsequent rejection (Figure 2a). Neither $8 \times 10^{6}$ tumor cells dying by expression of pro-apoptotic proteins, nor by expression of TNT prevented this natural allogeneic rejection from occuring (Figures $2 b-d$ ).
Recent findings from our group showed that high amounts $\left(16 \times 10^{6}\right)$ of cells dying in the absence of ROS production, are ignored by the immune system. Mice treated in this way show the same phenotype as naive mice with regard to the allogeneic rejection observed when challenged with viable B644 cells. ${ }^{8}$ As lower amounts $\left(8 \times 10^{6}\right)$ of dying cells failed to recapitulate this effect, we suggest that an overwhelming of the animal's local clearance capacity is of minor importance in determining the outcome of the allogeneic rejection. Therefore, we wondered whether an even smaller dose $\left(2 \times 10^{6}\right)$ of UVB-irradiated B644 cells, resembling a control for classical apoptosis, would be able to induce allogeneic rejection of a secondary challenge. Mice immunized in such a manner showed increased rejection of the tumor compared with naive mice (Supplementary Figure S4A). In contrast, the same amount of cells failed to induce a protective immune response in MHC-matched mice (Supplementary Figure S5A). Higher $\left(8 \times 10^{6}\right)$ amounts of melanoma cells dying by UVB-induced apoptosis, however, did protect mice from a challenge with viable cells under syngeneic conditions (Supplementary Figure S5A).

As the allogeneic setup did not allow us to distinguish whether the challenge with viable tumor cells was rejected because of the allogeneic rejection, the immunogenicity of cell death or a combination of both, we proceeded to investigate the immunological consequences of immunization with melanoma cells dying by the same death stimuli, but in a syngeneic background. All the modes of cell death tested conveyed significant protection against a secondary challenge with viable cells (Figures $3 a-c)$. However, the protection mediated by immunization with cells expressing TNT was significantly less pronounced than an immunization with cells dying due to the expression of pro-apoptotic proteins (Figure 3d).

In the mice immunized with cells dying by overexpression of cytotoxic proteins, we did not observe a dose-dependent effect as we had seen for the immunization with cells dying by UVB irradiation (Supplementary Figure S5A), because immunization with low doses of cells undergoing cell death also provided protection (Supplementary Figures S5B and D). This indicates that conditional expression of all the three death stimuli induced immunogenic cell death and that the effects were not caused by an overwhelming of clearance capacity.

We further analyzed whether the protection observed in the syngeneic background was mediated by lymphocytes. To do this, we isolated splenocytes from immunized MHC-matched mice and transferred these into recipient mice, which were subsequently challenged with viable B644 melanoma cells. We observed that the transfer of $20 \times 10^{6}$ splenocytes from mice that had received an immunization with dying cells endowed protective immunity (Figure 4). The protective effect was strongest for splenocytes derived from mice that had been immunized with cells dying by expression of revC3 and tBid, and was also visible for cells dying by expression of TNT.

To explain the outcomes of the various immunization experiments, we analyzed the dying cells for the release of potential 'find me' signals and danger-associated molecular patterns. As marked changes in mitochondrial membrane potential were apparent between the different forms of cell death, we checked for differences in ATP release. We 

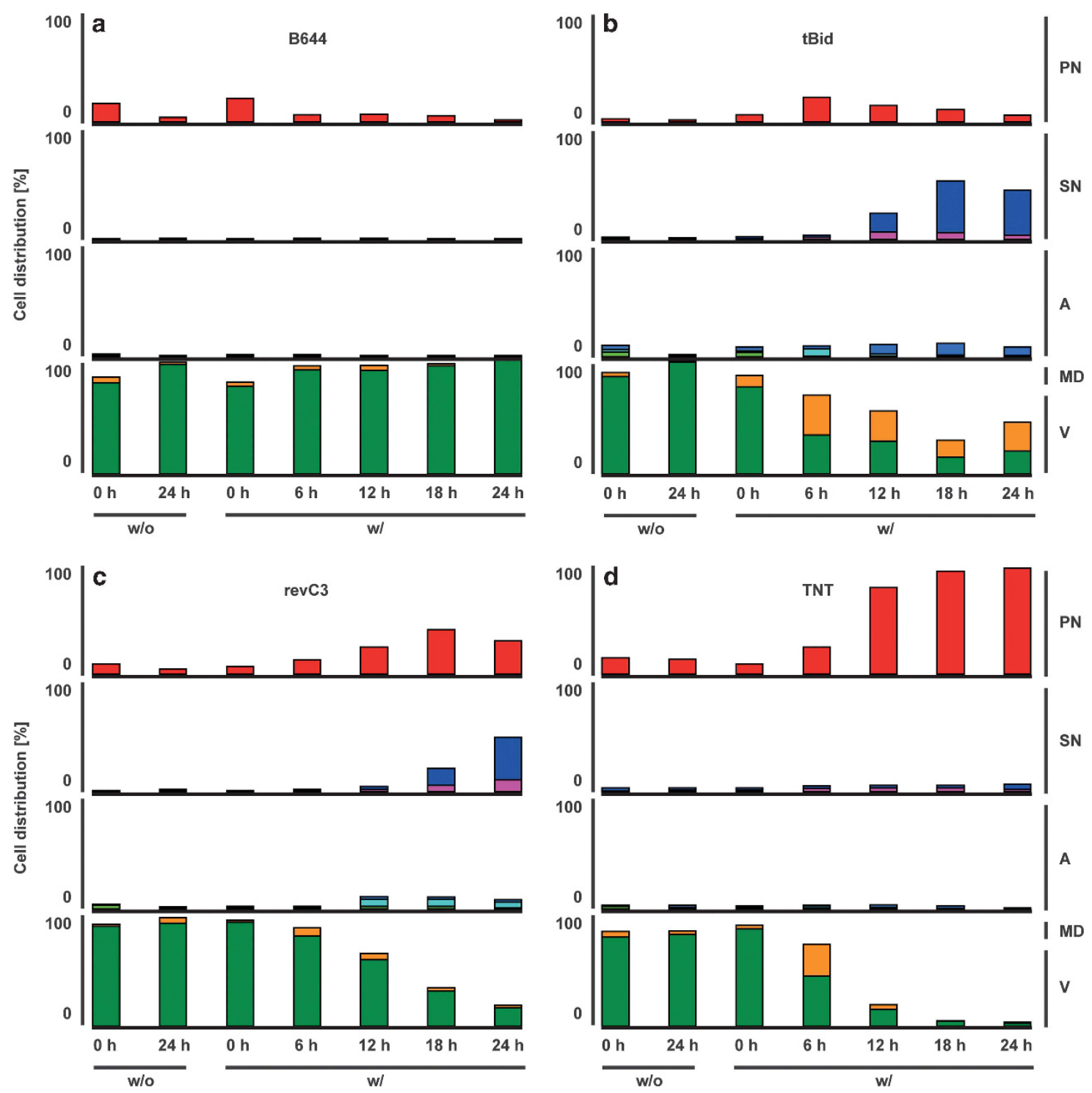

Figure 1 Flow cytometric characterization of the B16F10 melanoma suicide switch. B644 melanoma cells and its derivatives were either incubated in the presence (w/) or absence (w/o) of $5 \mu \mathrm{g} / \mathrm{ml}$ doxycycline. (a) B644 cells without expression of cytotoxic proteins, (b) B644 cells conditionally expressing tBid, (c) B644 cells conditionally expressing revC3 and (d) B644 cells conditionally expressing TNT. The cells were characterized as follows: viable (V), dark green; negative for phosphatidylserine, negative for propidium iodide, normal morphology and high for DilC1(5). Mitochondrial dysfunction (MD), orange; negative for phosphatidylserine, negative for propidium iodide, normal morphology, however, low for DilC1(5). Apoptotic cells (A). Early apoptotic, light green; normal morphology, positive for phosphatidylserine, negative for propidium iodide and high for DilC1(5); apoptotic, turquois; normal morphology, positive for phosphatidylserine, negative for propidium iodide, normal morphology and low for DilC1(5); late apoptotic, dark blue; shrunken morphology, positive for phosphatidylserine, negative for propidium iodide and low for DilC1(5). Secondary necrotic cells (SN), pink; shrunken morphology, positive for phosphatidylserine, positive for propidium iodide, low for DilC1(5) and high for Hoechst 33342; late secondary necrotic, purple; shrunken morphology, positive for phosphatidylserine, positive for propidium iodide, low for DilC1(5) and low for Hoechst 33342. Primary necrotic or early secondary necrotic cells (PN), red; shrunken morphology, positive for phosphatidylserine, highest positivity for propidium iodide, low for DilC1(5) and high for Hoechst 33342

observed that UVB-irradiated cells and cells dying by expression of TNT showed significantly higher and earlier secretion of ATP compared with cells dying by expression of revC3 and tBid (Figure 5a).

We additionally looked for danger-associated molecular pattern release during cell death. Huge quantities of heatshock protein 90 (HSP90) were present in the supernatant of cells dying by expression of revC3 and tBid, respectively, with the latter showing HSP90 release as early as $6 \mathrm{~h}$ after the induction of cell death. HSP90 was also detected in the supernatants of UVB-irradiated cells and cells expressing TNT, however, not before $24 \mathrm{~h}$ after the induction of cell death (Figure $5 \mathrm{~b}$ ). In line with this finding, cells dying by expression of TNT showed significantly less release of HMGB1 than cells dying by expression of $\mathrm{tBid}$ and revC3, respectively, which showed an early release of high amounts of HMGB1 (Figure 5c).

To further characterize the immunomodulatory potential of the various forms of cell death, we co-cultivated bonemarrow-derived macrophages (BMDM) with dying cells. The 

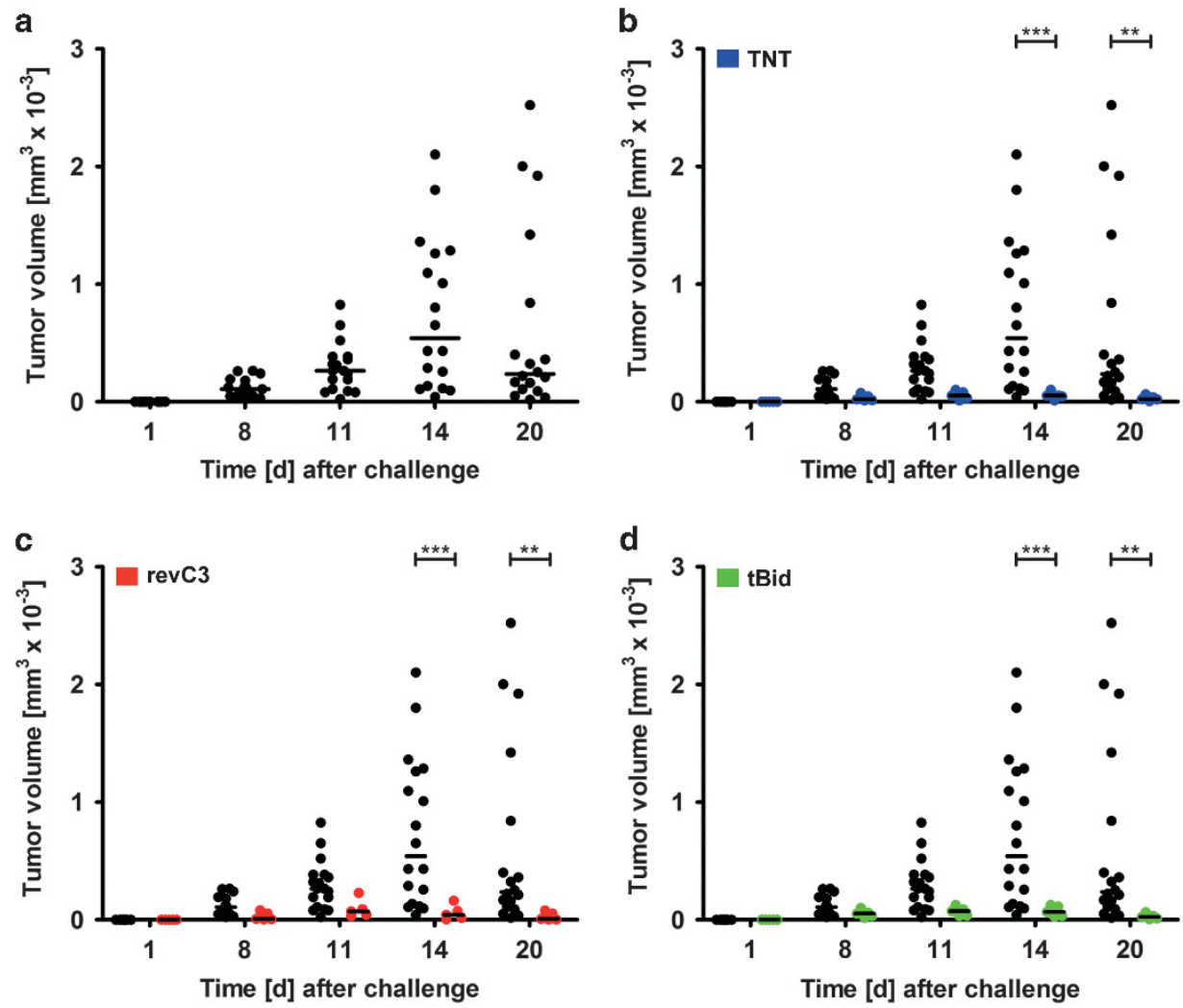

Figure 2 Allogeneic B16F10 melanoma growth in Balb/c mice. (a) Tumor growth in naive mice; (b) tumor growth in naive mice (black) and mice immunized with $8 \times 10^{6}$ cells dying by expression of TNT (blue); (c) tumor growth in naive mice (black) and mice immunized with $8 \times 10^{6}$ cells dying by expression of revC3 (red); and (d) tumor growth in naive mice (black) and mice immunized with $8 \times 10^{6}$ cells dying by expression of tBid (green). Plotted are single values, the horizontal line represents median tumor size. A two-way ANOVA analysis with Bonferroni correction for multiple comparisons was performed. ${ }^{* *}$ and ${ }^{* *}$ indicate statistical significance with $P$-values of 0.01 and 0.001 , respectively

concentrations of cytokines in the supernatant were evaluated $18 \mathrm{~h}$ after the induction of cell death. It must be noted that B16F10 melanoma cells express and secrete IL-6 per se. ${ }^{16}$ However, no differences in the secretion of IL- 6 was detected among the cell lines utilized for immunization, indicating that the differences observed are unique for the mode of cell death (Supplementary Figure S6). The concentrations of the cytokines IL-1 $a$, IL-1 $\beta$, IFN $\beta$, IL-10 and IL-27 were significantly lower in the supernatants of BMDM co-incubated with cells dying by expression of TNT compared with cells dying by expression of revC3 and $\mathrm{tBid}$, respectively (Figure 6). No differences were observed in the concentration of IL-12, although UVB-irradiated cells reduced the secretion of this cytokine (Figure 6).

\section{Discussion}

We have shown that our established six-parameter classification of cell death for flow cytometry recapitulates the microscopic changes accompanying cell death. ${ }^{17}$ This allows us to monitor morpho-physiological changes during cell death over an extended period of time.

Our findings further show that immunization with melanoma cells dying either by expression of tBid, revC3 or TNT fails to overcome allogeneic rejection of a secondary challenge with viable tumor cells. Immunization with these modes of cell death endowed protection in syngeneic conditions. As shown by splenocyte transfer, protection relied on lymphocytes, indicating establishment of immune memory. Melis and colleagues obtained similar results for revC3-induced cell death (Melis, 2013), ${ }^{18}$ but no studies have been performed on cell death caused by expression of tBid or the mycobacterial toxin TNT, respectively. We show that cell death induced by expression of TNT behaves in a similar manner as the UVBinduced apoptosis with regard to the release of 'ATP' and DAMPs (HMGB1, HSP90). ATP has versatile functions in the clearance of dying cells and is not only considered to be an important 'find me' signal released from apoptotic cells, ${ }^{19}$ but also a hallmark feature of immunogenic apoptosis. ${ }^{20}$ HMGB1 was initially characterized as a protein released from primary necrotic cells; ${ }^{21}$ however, more recent findings indicate that it is more efficiently released from cells undergoing secondary necrosis. $^{22}$

When co-incubated with BMDM, cells dying due to expression of TNT stimulated cytokine secretion to a far lesser extent than cells dying by the expression of revC3 and tBid, respectively. This was especially pronounced for the cytokines IL1- $a$, IL-10 and IL-27. Likewise, protection in the syngeneic tumor challenge model was significantly less pronounced in mice that had been immunized before with cells dying by the expression of TNT. This challenges the classical paradigm that necrotic cells are always inflammatory and induce robust immune responses. 

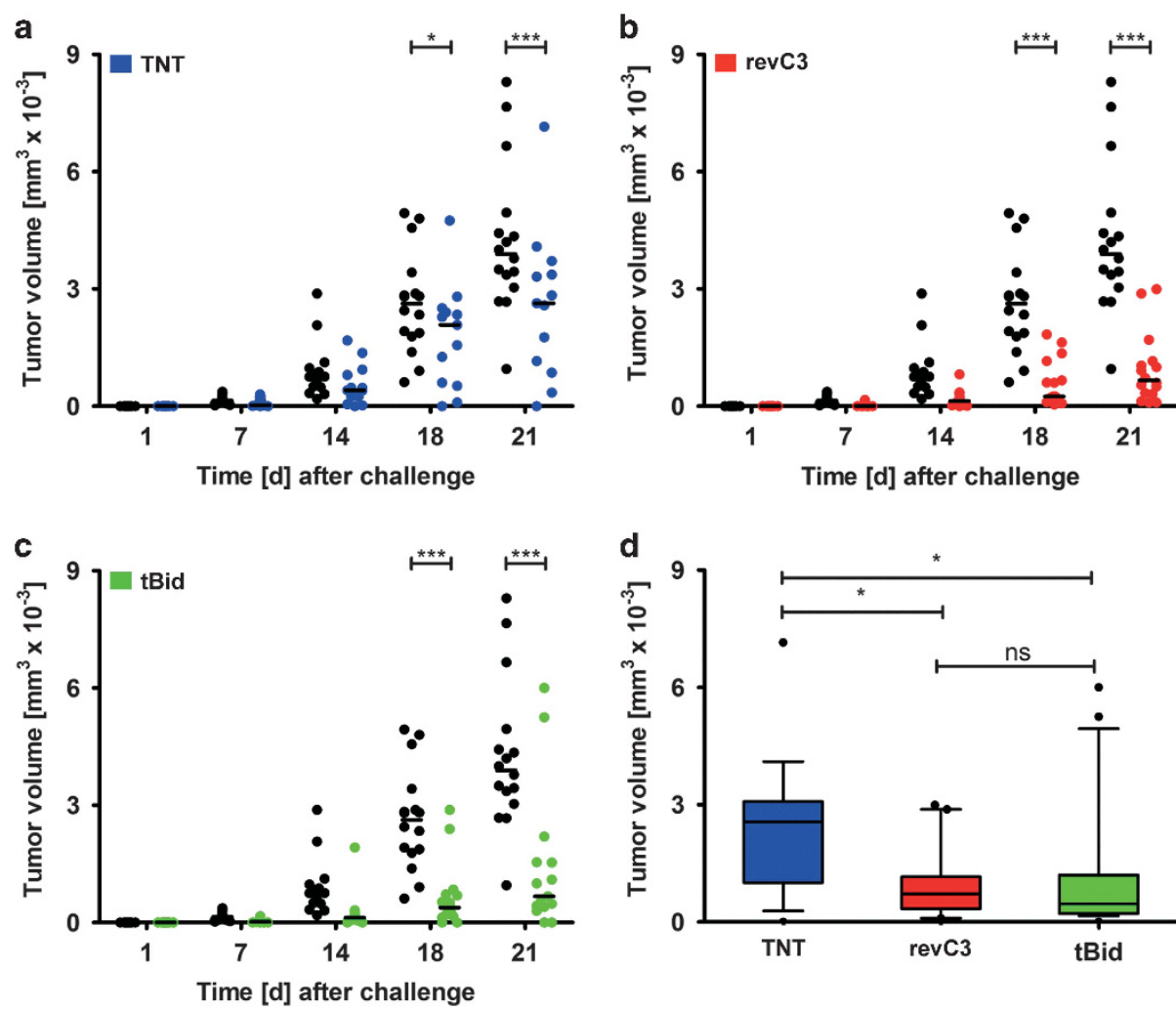

Figure 3 Syngeneic B16F10 melanoma growth in C57BL/6 mice. (a) Tumor growth in naive mice (black) and mice immunized with $8 \times 10^{6}$ tumor cells dying by expression of TNT (blue); (b) tumor growth in naive mice (black) and mice immunized with $8 \times 10^{6}$ tumor cells dying by expression of revC3 (red); (c) tumor growth in naive mice (black) and mice immunized with $8 \times 10^{6}$ tumor cells dying by expression of tBid (green); and (d) combined data of tumor growth in mice immunized with $2 \times 10^{6}$ or $8 \times 10^{6}$ tumor cells dying by the indicated stimulus. Plotted are single values, the horizontal line represents median tumor size. For multiple time points, a two-way ANOVA analysis with Bonferroni correction for multiple comparisons was performed. For (d), a similar one-way ANOVA analysis was performed. * and ${ }^{* \star *}$ indicate statistical significance with $P$-values of 0.05 and 0.001 , respectively; ns, not significant

Our data further suggest that secretion of ATP in the temporary absence of DAMPs induces a silent reaction (UVB, TNT), whereas the co-presence of these signals stimulates inflammatory responses (revC3, tBid). On the basis of these findings, we propose that the secretion of ATP attracts phagocytes which remove dying cells. In contrast, the simultaneous secretion of both ATP and DAMPs attracts and activates phagocytes to clear dying or dead cells in an inflammatory manner (Figure 7). Therefore, we hypothesize that ATP is not the determinant that controls whether cells are cleared in an inflammatory manner or not, but that it rather defines the time point of phagocyte attraction toward the site of cell death.

Much effort has been put into investigating how apoptotic cells are cleared. However, significantly less is known about the clearance of necrotic cell material. Several reports indicate that necrotic cells are not as potent inducers of inflammation and immunity than previously thought. Brouckaert et al. ${ }^{23}$ found that necrotic cells were unable to stimulate an inflammatory response on phagocytosis by macrophages. They further observed that necrotic cell death, induced by a combined treatment of staurosporine and oligomycin, dampened inflammatory responses as well as apoptotic cells. ${ }^{24}$ Similarly, LPS-stimulated macrophages and monocytes 'fed' with primary necrotic peripheral blood lymphocytes showed decreased secretion of TNFa and increased secretion of IL-10. ${ }^{25}$ Another report stated that phagocytosis of necrotic cells was unable to stimulate an inflammatory response in macrophages; however, they were able to amplify cytokine secretion in response to LPS. ${ }^{26}$ In a systemic study analyzing the adjuvant properties of various forms of cell death, oligomycin-induced necrosis failed to recapitulate the adjuvant effect of apoptotic cells, thereby successfully linking local inflammation and systemic immunity. ${ }^{27}$ In another recent study, phagocytosis of cells that had undergone necroptosis failed to induce inflammatory cytokine production. ${ }^{28}$

Further, our findings indicate a fundamental difference in how dying cells modulate the immune system under syngeneic and allogeneic conditions. Lower amounts of dying cells tend to favor an allogeneic rejection, whereas high amounts of cells dying without the production of cellular ROS failed to stimulate the allogeneic rejection of a secondary challenge with viable cells and rather led to a rejection similar to that observed for naive mice. ${ }^{8}$ In the syngeneic setting, low amounts of cells undergoing classical apoptosis are silently cleared without inducing immune responses, whereas intermediate amounts induce a robust immune response. This may be attributed to an overloading of the local clearance capacity, a factor apparently of lesser importance in allogeneic conditions. 

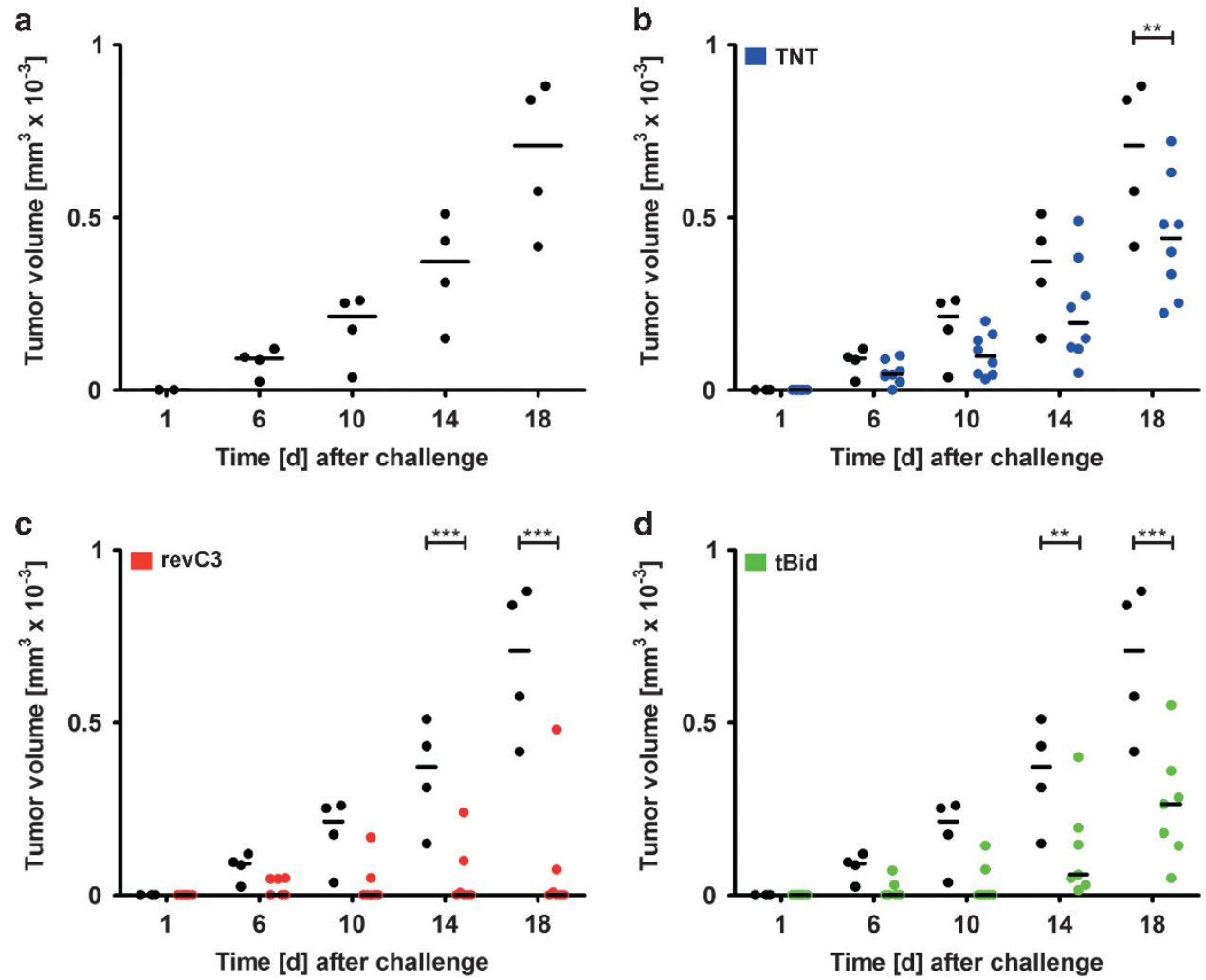

Figure 4 B16F10 melanoma growth after splenocyte transfer from immunized mice. Mice from Figure 5, which showed the smallest tumor growth, were killed and $20 \times 10^{6}$ splenocytes were injected in naive C57BL/6 mice. Two days after adoptive transfer, these mice were challenged with $0.5 \times 10^{6}$ viable tumor cells. (a) Tumor growth in mice that received splenocytes from non-immunized mice; (b) tumor growth in mice that received splenocytes from non-immunized mice (black), and mice that received splenocytes from mice which were immunized with cells dying by expression of TNT (blue); (c) tumor growth in mice that received splenocytes from non-immunized mice (black), and mice that received splenocytes from mice that were immunized with cells dying by expression of revC3 (red); and (d) tumor growth in mice that received splenocytes from non-immunized mice (black), and mice that received splenocytes from mice that were immunized with cells dying by expression of tBid (green). Plotted are single values, the horizontal line represents median tumor size. A two-way ANOVA analysis with Bonferroni correction for multiple comparisons was performed. ${ }^{* *}$ and ${ }^{* * *}$ indicate statistical significance with $P$-values of 0.01 and 0.001 , respectively

With TNT being a mycobacterial toxin, its effects need to be considered in the context of a mycobacterial infection. It is an ongoing matter of debate whether MTB takes advantage of or tries to evade inflammatory host responses. ${ }^{29}$ Several studies have shown that MTB inhibits the induction of apoptosis ${ }^{30,31}$ and it is known that anti-apoptotic proteins like Mcl-1 (ref. 32) or A1 (refs 33,34 ) are upregulated upon MTB infection. Interestingly, induction of host cell apoptosis negatively correlates with virulence. ${ }^{35,36}$ Of particular interest is the finding that apoptotic bodies of MTB infected cells are taken up by dendritic cells and that mycobacterial antigens are cross-presented to cytotoxic T-lymphocytes. ${ }^{37}$ Likewise, treatment of mice with apoptotic bodies of MTB infected cells endowed protection against an MTB infection. ${ }^{38}$ Recent discoveries have shown that MTB induces an atypical cell death in infected host cells. This kind of cell death is characterized by the loss of mitochondrial membrane potential, depletion of ATP and the loss of plasma membrane integrity, thereby allowing cellular escape of MTB. ${ }^{15}$ TNT was identified to be a potent inducer of primary necrosis via depletion of $\mathrm{NAD}^{+}{ }^{10,11}$

These data suggest that MTB inhibits apoptosis to evade eradication and that it induces primary necrosis to promote spreading into the tissue with subsequent infection of other host cells. Thereby, TNT seems to be the main toxin of MTB, as strains genetically depleted of TNT failed to induce macrophage cell death. ${ }^{11}$ This renders TNT an interesting target for the therapy of tuberculosis: by targeting the protein's $\mathrm{NAD}^{+}$hydrolase activity, one could possibly prevent macrophage progression into primary necrosis. This might inhibit both spreading of the infection and counter the immune escape by allowing proper execution of apoptosis.

Remarkably, cells dying by expression of UVB and TNT failed to induce secretion of IL-27 from BMDM, whereas high concentrations of this cytokine were detected in the supernatants of BMDM stimulated with cells dying by expression of revC3 and tBid, respectively. IL-27 is an important factor linking innate and adaptive tumor immunity ${ }^{39}$ by not only enhancing natural killer cell-mediated killing of cancer cells, ${ }^{40}$ but also by fostering cytotoxic T-lymphocyte generation. ${ }^{41,42}$ In addition, IL-27 exerts direct anti-proliferative and antiangiogenic effects on melanoma cells. ${ }^{43,44}$ Interestingly, IL-27 signaling has also been reported to be an important factor in the control of MTB infections. ${ }^{45}$ It is, therefore, reasonable that MTB tries to evade forms of cell death that induce IL-27 secretion. 


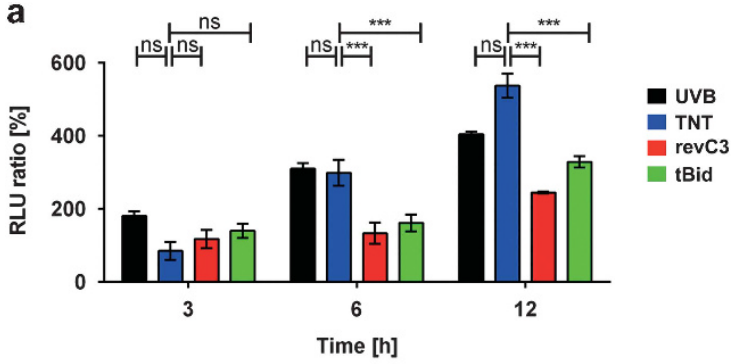

b
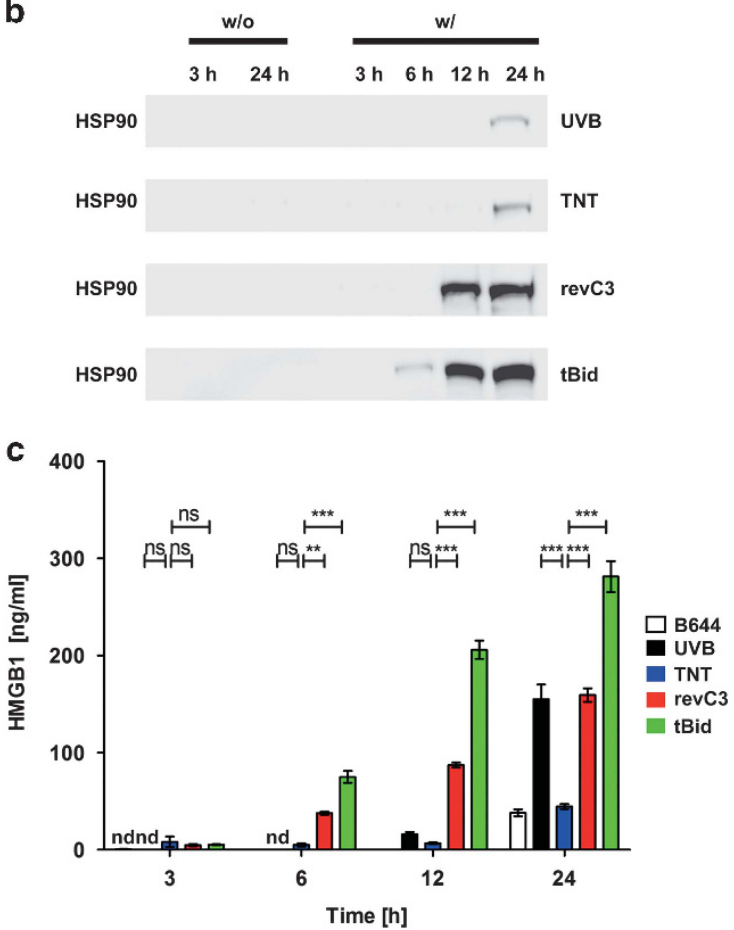

Figure 5 'Find me' signals and DAMPs released from dying cells. (a) ATP release by dying cells. ATP in the supernatant of dying cells was detected with a luminescence assay. Depicted is the luminescence ratio of cells dying by the respective stimulus and non-stimulated analogous cells $(N=4)$. Shown are means with their standard error of the mean. A two-way ANOVA analysis with Bonferroni correction for multiple comparisons was performed. ${ }^{* *}$ and ${ }^{* \star *}$ indicate statistical significance with $P$-values of 0.01 and 0.001 , respectively (b) HSP90 release by dying cells. Depicted is the luminescence signal in western blot analysis for the detection of HSP90. Supernatants of cells induced with death stimuli (w/) were analyzed 3, 6, 12 and $24 \mathrm{~h}$ after the induction of cell death. Analogous non-induced cells (w/o) served as negative control; (c) HMGB1 release by dying cells. HMGB1 in the supernatant of dying cells was detected with enzyme-linked immunosorbent assay $(N=4)$. Cell supernatants of viable melanoma cells served as negative control (only depicted for 0 and $24 \mathrm{~h}$ ). Shown are means with their standard error of the mean. 'ND' indicates non-detectable concentrations of HMGB1. A two-way ANOVA analysis with Bonferroni correction for multiple comparisons was performed. ${ }^{* *}$ and ${ }^{* \star *}$ indicate statistical significance with $P$-values of 0.01 and 0.001 , respectively; ns, not significant

Our findings indicate that the release of 'find me' signals like ATP in the temporal absence of DAMPs like HSP9O and HMGB1 is associated with poor immune responses, whereas the concurrence of both signals induces an inflammatory response and antitumor immunity (Figure 7). Taken together, our data support a steadily increasing number of studies demonstrating that the classical paradigm of apoptosis as an exclusively anti-inflammatory form of cell death and necrosis as a condition causing inflammation is not applicable in all contexts of cell death. Instead it might rather be decisive when and under which conditions phagocytes are recruited to the site of cellular demise.

\section{Materials and Methods}

Reagents and molecular probes. Gibco Dulbecco's modified Eagle's medium (DMEM), Gibco RPMI 1640, Gibco G418 sulfate, Gibco penicillinstreptomycin, Gibco glutamine, Gibco puromycin dihydrochloride, Gibco trypsin EDTA (10x), MitoProbe 1,10,3,3,30,30-hexamethylindodicarbocyanine iodide dye (DilC1(5)) and Molecular Probes Hoechst 33342 were bought from Thermo Scientific (Darmstadt, Germany). Recombinant chicken annexin A5 (AxV) was supplied by Responsif (Erlangen, Germany). Doxycycline hydrochloride, propidium iodide (PI) and the 'FluoroTag FITC Conjugation Kit' for conjugation of AxV with FITC were obtained from Sigma-Aldrich (Darmstadt, Germany). Ringer's solution was from Delta Select (Pfullingen, Germany) and Biochrom tetracycline-free fetal bovine serum albumin (FBS) was purchased from Merck-Millipore (Darmstadt, Germany).

Cell lines and culture conditions. The C57BL/6 mouse-derived melanoma cell line B16F10 (haplotype H2b) was purchased from the ATCC (\#CRL-6475) and the cell line and its derivatives were propagated in DMEM supplemented with $10 \%\left(\mathrm{v} / \mathrm{v}\right.$ ) FBS and penicillin-streptomycin (D10) at $37^{\circ} \mathrm{C}$ in a $5 \% \mathrm{CO}_{2}$ atmosphere. The construction of the suicide switch and its components have been thoroughly described elsewhere. ${ }^{8}$

Cell death induction. For expression of the cytotoxic proteins tBid, revC3 and TNT, cell death was induced by adding doxycycline to the cells in vitro $(5 \mu \mathrm{g} / \mathrm{ml})$. Classical apoptosis was induced by irradiation of the cell lines B644 with ultraviolet light type B (UVB) at $1.5 \mathrm{~mJ} / \mathrm{cm}^{2} / \mathrm{s}$. For in vivo experiments, the cells were induced with $5 \mu \mathrm{g} / \mathrm{ml}$ doxycycline or a corresponding dose of UVB irradiation in vitro. Implantation into the animals was performed $4 \mathrm{~h}$ post induction before any morphological changes were visible.

Multiparameter classification of cell death by flow cytometry. For the morpho-physiological characterization by flow cytometry, B644 cells and its derivatives were cultured in 12-well plates at 200000 cells per $2 \mathrm{ml} \mathrm{D10}$. At the time points indicated, supernatants containing the dead cells were collected into polypropylene tubes. A trypsin-EDTA solution was added to the wells for 10-15 min at room temperature to detach any remaining adherent cells. Detached cells were collected by adding D10 and combined with their corresponding supernatant fraction. Finally, cells were centrifuged at $300 \times g$ for $5 \mathrm{~min}$ and resuspended in the appropriate medium. The cell death characterization method analyzing size, granularity, phosphatidylserine exposure, plasma membrane integrity, mitochondrial membrane potential and DNA content in a one-tube-measurement has been thoroughly described elsewhere. ${ }^{16}$ Briefly, collected cells were resuspended in approximately $100 \mu \mathrm{l}$ of backflow and $400 \mu \mathrm{l}$ of freshly prepared four-color staining solution containing $1 \mathrm{mg} / \mathrm{ml}$ AxV-FITC, $100 \mathrm{ng} / \mathrm{ml}$ PI, $10 \mathrm{nM}$ DilC1(5), $1 \mu \mathrm{g} / \mathrm{ml}$ Hoechst 33342 in Ringer's solution were added. After incubation for $30 \mathrm{~min}$ at room temperature, samples were analyzed with a Gallios flow cytometer (Beckman Coulter, Krefeld, Germany). Electronic compensation was applied to reduce bleedthrough fluorescence and data analysis was performed with the software Kaluza 1.3 (Beckman Coulter).

Multiparameter classification of cell death by live cell imaging. Lab-Tek chamber slides (Thermo Fisher Scientific, Darmstadt, Germany) were coated with poly-L-lysine and cells were seeded at a concentration of 50000 cells per well in $800 \mu \mathrm{l}$ of D10 and incubated for $18 \mathrm{~h}$ at $37^{\circ} \mathrm{C}$ and $5 \% \mathrm{CO}_{2}$. One hour before the addition of doxycycline $(5 \mu \mathrm{g} / \mathrm{ml}), 2 \mathrm{mg} / \mathrm{ml} \mathrm{AxV-FITC,} 400 \mathrm{ng} / \mathrm{ml} \mathrm{Pl}$, $40 \mathrm{nM}$ DilC1(5) and $0.1 \mu \mathrm{g} / \mathrm{ml}$ Hoechst 33342 were added to the medium. These concentrations proved to be nontoxic during the observation period (18 h). Live cell imaging was performed with an Axio Observer.Z1 (Zeiss, Oberkochen, Germany) microscope equipped with two incubation chambers (PM S1, XLmultiS1 (both Zeiss)) for live cell imaging under cell culture conditions $\left(37^{\circ} \mathrm{C}, 5 \% \mathrm{CO}_{2}\right)$. The 

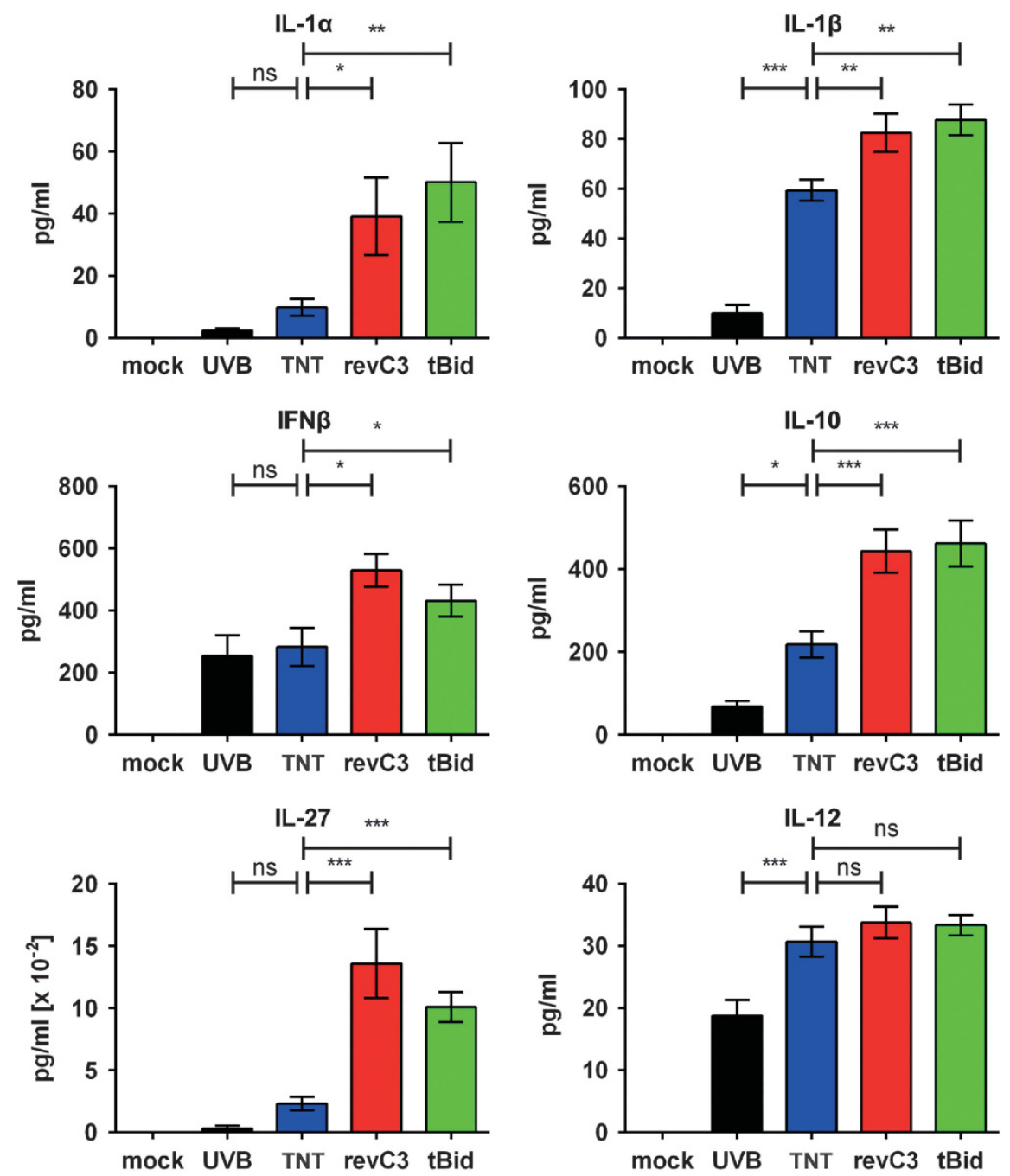

Figure 6 Cytokine secretion of bone-marrow-derived macrophages (BMDM) in response to co-incubation with dying cells. BMDM were co-cultivated for $18 \mathrm{~h}$ with cells dying by the indicated stimuli. The respective supernatants were analyzed for the cytokines IL-1 $\alpha$, IL-1 $\beta$, IFN $\beta$, IL-10, IL-27, IL-6 and IL-12 ( $N=8$ ). Shown are means with their standard error of the mean. A one-way ANOVA analysis with Bonferroni correction for multiple comparisons was performed. * ${ }^{* *}$ and ${ }^{* *}$ indicate statistical significance with $P$-values of $0.05,0.01$ and 0.001 , respectively; ns, not significant

instruments were operated with the software ZEN pro 2012 (Zeiss). Photos were post-processed with Photoshop CS 6 (Adobe, Dublin, Ireland).

ATP detection. ATP in the supernatant of dying or dead cells was detected with the 'Luminescent ATP Detection Assay Kit' (Abcam, Milton, United Kingdom) according to the manufacturer's instructions. Briefly, 12-well plates containing the dying or dead cells were centrifuged at $300 \times g$ for $5 \mathrm{~min}$ and $100 \mu \mathrm{l}$ of supernatant was carefully removed and added to a plate filled with $50 \mu \mathrm{l}$ of lysis buffer to stabilize ATP and inhibit degradation. These plates were immediately kept at $-80^{\circ} \mathrm{C}$ and luminescence detection was performed within 1 week on a Centro LB 960 (Berthold, Bad Wildbad, Germany) luminometer. For operation, the software MikroWin 20004.29 (Berthold) was utilized. Depicted is the ratio of the luminescence signal derived from supernatants of dying or dead cells and analogous supernatants of living cells.

Western blot analysis. Twelve-well plates containing cells treated with the respective death stimulus were centrifuged at $300 \times g$ for 5 min at the time points indicated and $200 \mu \mathrm{l}$ supernatant was carefully removed and immediately stored at $-80^{\circ} \mathrm{C}$ until western blot analysis was performed. Samples were defrosted on ice, proteins were precipitated with trichloroacetic acid and the pellet was washed with acetone/methanol. The pellet was resuspended in $40 \mu \mathrm{l}$ of $1 \times$ sample buffer and proteins were separated on a 4-20\% TRIS-glycine SDS-PAGE (VWR, Darmstadt, Germany) under denaturating conditions. Following this, proteins were blotted on a polyvinylidenfluorid membrane (Macherey-Nagel, Düren, Germany). HSP90 was detected with the antibody combination $\alpha$-HSP90 IgG (New England BioLlbs, Frankfurt, Germany, 4874) and $\alpha$-rabbit IgG conjugated with horseradish peroxidase (DakoCytomation, Hamburg, Germany, P0448) following the manufacturers' instructions. Luminescence detection was performed with a Celvin S (Biostep, Burkhardtsdorf, Germany) and the software SnapAndGo 1.6.1 (Biostep).

Enzyme-linked immunosorbent assay. Twelve-well plates containing cells treated with the respective death stimulus were centrifuged at $300 \times g$ for 5 min at the time points indicated and $200 \mu \mathrm{l}$ supernatant was carefully removed and immediately stored at $-80^{\circ} \mathrm{C}$ until enzyme-linked immunosorbent assay was performed. HMGB1 quantification was performed with the HMGB1 ELISA Kit II (IBL International, Hamburg, Germany) following the manufacturer's instructions. The absorbance was measured with an Infinite 200 PRO plate reader (TECAN, Crailsheim, Germany) and the software i-control 1.10 (TECAN).

Co-incubation of dying or dead cells with bone-marrow-derived macrophages. Bone marrow cells were isolated from $\mathrm{C} 57 \mathrm{BL} / 6$ mice and plated in RPMI 1640 supplemented with 10\% (v/v) FCS, 1\% (v/v) penicillin-streptomycin 
a

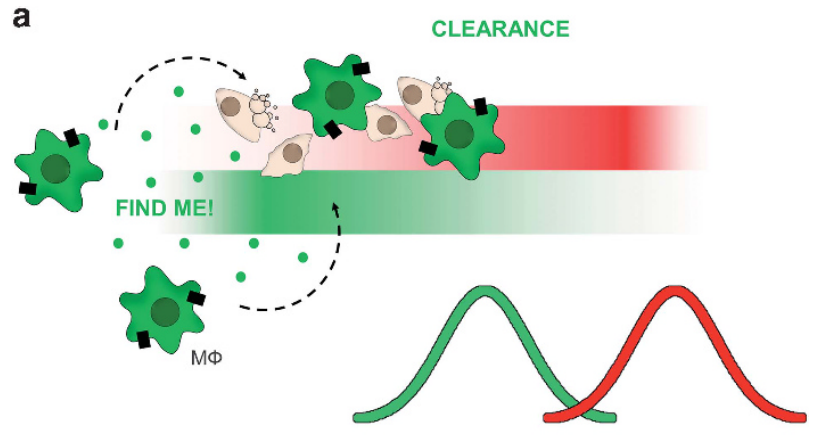

b
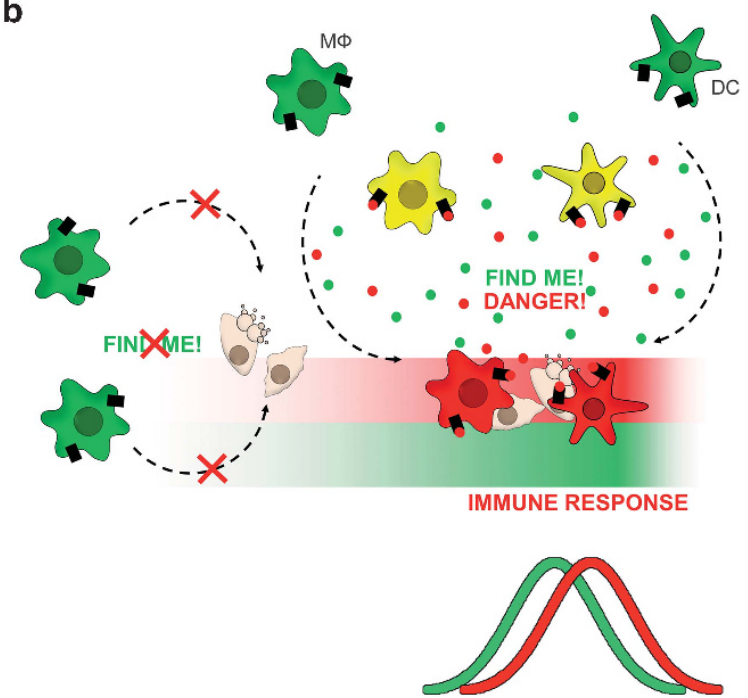

Figure 7 Conceptual model of the interplay of 'find me' signals and DAMPs in the clearance of dying cells. (a) Immunologically silent cell death. If 'find me' signals (i.e. ATP, green bar) are released from dying cells before the release of DAMPs (i.e. HMGB1, red bar), dying cells are cleared silently by resident macrophages. (b) Immunogenic cell death. The simultaneous release of 'find me' signals (i.e., ATP, green bar) and DAMPs (i.e., HMGB1, red bar) also recruits phagocytes (macrophages, dendritic cells) to the site of cell death. However, on their way to the prey or when arriving at the site of cell death, they will be activated by the inflammatory components released from the dying cells, thus exerting a proinflammatory phenotype

and $1 \%\left(\mathrm{v} / \mathrm{v}\right.$ ) glutamine (R10). The cells were cultivated for $24 \mathrm{~h}$ at $37{ }^{\circ} \mathrm{C}$ under $5 \%$ $\mathrm{CO}_{2}$. Non-adherent cells were collected and adjusted to 100.000 cells per milliliter in R10 supplemented with $10 \%$ conditioned L929 medium. A total $1 \times 10^{5}$ cells were added to each well of a 24-well plate and cultured for 3 days at $37{ }^{\circ} \mathrm{C}$ under $5 \%$ $\mathrm{CO}_{2}$. After $72 \mathrm{~h}, 0.5 \mathrm{ml}$ of R10 supplemented with $10 \%$ conditioned L929 medium were added and the cells were incubated for another 3 days. Cell supernatants were removed and $5 \times 10^{5}$ cells or respective controls in D10 with $5 \mu \mathrm{g} / \mathrm{ml}$ doxycycline were added for induction of cell death in the presence of macrophages. The cells were incubated for $18 \mathrm{~h}$, centrifuged at $300 \times g$ for $5 \mathrm{~min}$ and $200 \mu \mathrm{l}$ of supernatant was collected and stored at $-80^{\circ} \mathrm{C}$ until cytokine measurement. Cytokine concentrations were determined with LEGENDplex Mouse Inflammation Panel (13-plex; Biolegend, Fell, Germany) according to the manufacturers' instructions. The analysis was performed with a CytoFLEX flow cytometer (Beckman Coulter) and the data were processed with the software Cytexpert 1.2.8.0 (Beckman Coulter).

Immunization, tumor challenge and spleenocyte transfer. The mice were purchased from The Jackson Laboratory (Sulzfeld, Germany) and kept on a standard diet with drinking water available ad libitum. The experiments were conducted according to the European principles and local guidelines for care and use of laboratory animals at the Institute of Cell Biology, National Academy of Sciences of Ukraine, Lviv and the Frantz-Penzoldt-Centre, Erlangen.

The general immunization scheme is depicted in Supplementary Figure S7.

(1) The allogeneic tumor challenge model for analyzing the immunomodulatory effects of dying tumor cells consisted of the host mouse (BALB/c, MHC haplotype $\mathrm{H} 2 \mathrm{~d}$ ) and B16F10 melanoma cells derived from C57BL/6 mice (MHC haplotype H2b). Specified amounts of cells induced to die by the respective stimulus were subcutaneously (s.c.) implanted in $500 \mu$ of Ringer's solution in the right flank using a syringe with a $25 \mathrm{G}$ needle. Eight to 10 days after immunization, the mice were challenged s.c. with $2 \times 10^{6}$ viable B644 cells in the opposite flank.

(2) The syngeneic tumor challenge model for analyzing the immunomodulatory effects of dying tumor cells consisted of the host mouse (C57BL/6, MHC haplotype $\mathrm{H} 2 \mathrm{~b}$ ) and B16F10 melanoma cells derived from C57BL/6 mice (MHC haplotype H2b). Specified amounts of cells induced to die by the respective stimulus were implanted in $500 \mu \mathrm{l}$ of Ringer's solution (s.c.) in the right flank using a syringe with a $25 \mathrm{G}$ needle. Eight to 10 days after immunization, the mice were challenged s.c. with $1 \times 10^{6}$ viable B644 cells in the opposite flank.

(3) The transfer of splenocytes was performed as follows: mice from the syngeneic tumor challenge model showing the smallest tumor size after 21 days were killed and splenocytes were isolated. A total $20 \times 10^{6}$ splenocytes in a volume of $200 \mu \mathrm{l}$ of Ringer's solution were injected with a $25 \mathrm{G}$ needle into naive C57BL/6 mice. Two days after splenocyte transfer, the animals were s.c. challenged with $5 \times 10^{5}$ viable B16F10 melanoma cells and tumor size was monitored.

The growth of solid melanoma tumors was registered by direct measurement of width, height and depth of the black subcutaneous protuberance with a caliper for up to a maximum of 21 days. In accordance with the guidelines for the welfare of animals in experimental neoplasia, the animal was killed if the mouse tumor volume exceeded more than $10 \%$ of the host's bodyweight.

\section{Conflict of Interest}

The authors declare no conflict of interest.

Acknowledgements. This work was supported by the following programs of the 'German Research Foundation' (DFG): SFB-643 (MH), SFB-1181 (LEM, MH), SPP1681 (RPF) and the doctoral training programs GK1660 (CM, MH) and SFB-643 (RAC, MJP, MH). GS was supported from the IMI funded research project BTCure. $\mathrm{MH}$ got additional support from the KFO257 of the University of Erlangen-Nuremberg. RB and TD were supported by the National Academy of Sciences, Ukraine. L929 conditioned medium was a kind gift of Dr. N Ipseiz. Flow cytometry was performed in cooperation with the flow cytometry/cell sorting core facility of the SFB-643. We thank Professor Dr. M Niederweis for supplying the CDNA of TNT and A Karmash for excellent technical assistance. We further thank Dr. B Frey and Dr. U Gaipl for help with the establishment of methods and their valuable advice.

\section{Author contributions}

CM, RAC, MJP and LEM planned and performed most of the in vitro and in vivo experiments, conducted the data analysis and wrote the manuscript. TD, MDL, RB and $\mathrm{SC}$ performed some in vivo experiments and conducted the data analyses. CM, LEM, MH and RAC performed the flow cytometry analysis. CM, RPF and MJP performed and evaluated live fluorescence microscopy. RB, GS and CA provided scientific input and wrote the manuscript. LM, MH and CB supervised the project, planned and conducted the experiments, made data analysis and wrote the manuscript. All the authors read and approved the manuscript.

1. Elmore S. Apoptosis: a review of programmed cell death. Toxicol Pathol 2007; 35: 495-516.

2. Hochreiter-Hufford A, Ravichandran KS. Clearing the dead: apoptotic cell sensing, recognition, engulfment, and digestion. Cold Spring Harb Perspect Biol 2013; 5: a008748.

3. Nijhawan D, Honarpour N, Wang X. Apoptosis in neural development and disease. Annu Rev Neurosci 2000; 23: 73-87.

4. Opferman JT, Korsmeyer SJ. Apoptosis in the development and maintenance of the immune system. Nat Immunol 2003; 4: 410-415.

5. Munoz LE, Lauber K, Schiller M, Manfredi AA, Herrmann M. The role of defective clearance of apoptotic cells in systemic autoimmunity. Nat Rev Rheumatol 2010; 6: 280-289.

6. Gregory CD, Pound JD. Cell death in the neighbourhood: direct microenvironmental effects of apoptosis in normal and neoplastic tissues. J Pathol 2011; 223: 177-194. 
7. Maueroder C, Chaurio RA, Platzer S, Munoz LE, Berens C. Model systems for rapid and slow induction of apoptosis obtained by inducible expression of pro-apoptotic proteins. Autoimmunity 2013; 46: 329-335.

8. Chaurio RA, Munoz LE, Maueroder C, Janko C, Harrer T, Furnrohr BG et al. The progression of cell death affects the rejection of allogeneic tumors in immune-competent mice - implications for cancer therapy. Front Immunol 2014; 5: 560.

9. Green DR, Ferguson T, Zitvogel L, Kroemer G. Immunogenic and tolerogenic cell death. Nat Rev Immunol 2009; 9: 353-363.

10. Danilchanka O, Sun J, Pavlenok M, Maueroder C, Speer A, Siroy A et al. An outer membrane channel protein of Mycobacterium tuberculosis with exotoxin activity. Proc Natl Acad Sci USA 2014; 111: 6750-6755.

11. Sun J, Siroy A, Lokareddy RK, Speer A, Doornbos KS, Cingolani G et al. The tuberculosis necrotizing toxin kills macrophages by hydrolyzing NAD. Nat Struct Mol Biol 2015; 22: 672-678.

12. Walsh JG, Cullen SP, Sheridan C, Luthi AU, Gerner C, Martin SJ. Executioner caspase-3 and caspase-7 are functionally distinct proteases. Proc Natl Acad Sci USA 2008; 105 : 12815-12819.

13. Srinivasula SM, Ahmad M, MacFarlane M, Luo Z, Huang Z, Fernandes-Alnemri T et al. Generation of constitutively active recombinant caspases- 3 and -6 by rearrangement of their subunits. J Biol Chem 1998; 273: 10107-10111.

14. Kulms D, Zeise E, Poppelmann B, Schwarz T. DNA damage, death receptor activation and reactive oxygen species contribute to ultraviolet radiation-induced apoptosis in an essential and independent way. Oncogene 2002; 21: 5844-5851.

15. Lee J, Repasy T, Papavinasasundaram K, Sassetti C, Kornfeld H. Mycobacterium tuberculosis induces an atypical cell death mode to escape from infected macrophages. PLoS One 2011; 6: e18367.

16. Valles SL, Benlloch M, Rodriguez ML, Mena S, Pellicer JA, Asensi M et al. Stress hormones promote growth of B16-F10 melanoma metastases: an interleukin 6- and glutathionedependent mechanism. J Transl Med 2013; 11: 72.

17. Munoz LE, Maueroder C, Chaurio R, Berens C, Herrmann M, Janko C. Colourful death: sixparameter classification of cell death by flow cytometry - dead cells tell tales. Autoimmunity 2013; 46: 336-341.

18. Melis MH, Simpson KL, Dovedi SJ, Welman A, MacFarlane M, Dive $C$ et al. Sustained tumour eradication after induced caspase-3 activation and synchronous tumour apoptosis requires an intact host immune response. Cell Death Differ 2013; 20: 765-773.

19. Elliott MR, Chekeni FB, Trampont PC, Lazarowski ER, Kadl A, Walk SF et al. Nucleotides released by apoptotic cells act as a find-me signal to promote phagocytic clearance. Nature 2009; 461: 282-286.

20. Martins I, Wang Y, Michaud M, Ma Y, Sukkurwala AQ, Shen S et al. Molecular mechanisms of ATP secretion during immunogenic cell death. Cell Death Differ 2014; 21: 79-91.

21. Scaffidi $P$, Misteli T, Bianchi ME. Release of chromatin protein HMGB1 by necrotic cells triggers inflammation. Nature 2002; 418: 191-195

22. Urbonaviciute V, Furnrohr BG, Weber $\mathrm{C}$, Haslbeck M, Wilhelm S, Herrmann $\mathrm{M}$ et al. Factors masking HMGB1 in human serum and plasma. $J$ Leukoc Biol 2007; 81: 67-74.

23. Brouckaert G, Kalai M, Krysko DV, Saelens X, Vercammen D, Ndlovu MN et al. Phagocytosis of necrotic cells by macrophages is phosphatidylserine dependent and does not induce inflammatory cytokine production. Mol Biol Cell 2004; 15: 1089-1100.

24. Hirt UA, Leist M. Rapid, noninflammatory and PS-dependent phagocytic clearance of necrotic cells. Cell Death Differ 2003; 10: 1156-1164.

25. Bottcher A, Gaipl US, Furnrohr BG, Herrmann M, Girkontaite I, Kalden JR et al. Involvement of phosphatidylserine, alphavbeta3, CD14, CD36, and complement C1q in the phagocytosis of primary necrotic lymphocytes by macrophages. Arthritis Rheum 2006; $\mathbf{5 4}$ : 927-938.
26. Cocco RE, Ucker DS. Distinct modes of macrophage recognition for apoptotic and necrotic cells are not specified exclusively by phosphatidylserine exposure. Mol Biol Cell 2001; 12 919-930.

27. Shi Y, Zheng W, Rock KL. Cell injury releases endogenous adjuvants that stimulate cytotoxic T cell responses. Proc Natl Acad Sci USA 2000; 97: 14590-14595.

28. Wang Q, Ju X, Zhou Y, Chen K. Necroptotic cells release find-me signal and are engulfed without proinflammatory cytokine production. In Vitro Cell Dev Biol Anim 2015; 51 1033-1039.

29. Brighteni S, Lerm M. How mycobacterium tuberculosis manipulates innate and adaptive immunity - new views of an old topic. In: Cardona PJ (ed). Understanding Tuberculosis: Analyzing the Origin of Mycobacterium Tuberculosis Pathogenicity. InTech, Rijeka, Croatia, 2012, 207-234.

30. Briken V, Miller JL. Living on the edge: inhibition of host cell apoptosis by Mycobacterium tuberculosis. Future Microbiol 2008; 3: 415-422.

31. Blomgran R, Desvignes L, Briken V, Ernst JD. Mycobacterium tuberculosis inhibits neutrophil apoptosis, leading to delayed activation of naive CD4 T cells. Cell Host Microbe 2012; 11: 81-90.

32. Sly LM, Hingley-Wilson SM, Reiner NE, McMaster WR. Survival of Mycobacterium tuberculosis in host macrophages involves resistance to apoptosis dependent upon induction of antiapoptotic Bcl-2 family member Mcl-1. J Immunol 2003; 170: 430-437.

33. Kremer L, Estaquier J, Brandt E, Ameisen JC, Locht C. Mycobacterium bovis Bacillus Calmette Guerin infection prevents apoptosis of resting human monocytes. Eur J Immuno 1997; 27: 2450-2456.

34. Kausalya S, Somogyi R, Orlofsky A, Prystowsky MB. Requirement of A1-a for bacillus Calmette-Guerin-mediated protection of macrophages against nitric oxide-induced apoptosis. J Immunol 2001; 166: 4721-4727.

35. Dhiman R, Raje M, Majumdar S. Differential expression of NF-kappaB in mycobacteria infected THP-1 affects apoptosis. Biochim Biophys Acta 2007; 1770: 649-658.

36. Maiti D, Bhattacharyya A, Basu J. Lipoarabinomannan from Mycobacterium tuberculosis promotes macrophage survival by phosphorylating Bad through a phosphatidylinositol 3-kinase/Akt pathway. J Biol Chem 2001; 276: 329-333.

37. Schaible UE, Winau F, Sieling PA, Fischer K, Collins HL, Hagens K et al. Apoptosis facilitates antigen presentation to $\mathrm{T}$ lymphocytes through $\mathrm{MHC}-\mathrm{I}$ and $\mathrm{CD} 1$ in tuberculosis. Nat Med 2003; 9: 1039-1046.

38. Winau F, Weber S, Sad S, de Diego J, Hoops SL, Breiden B et al. Apoptotic vesicles crossprime CD8 T cells and protect against tuberculosis. Immunity 2006; 24: 105-117.

39. Murugaiyan G, Saha B. IL-27 in tumor immunity and immunotherapy. Trends Mol Med 2013 19: $108-116$.

40. Liu L, Wang S, Shan B, Shao L, Sato A, Kawamura K et al. IL-27-mediated activation of natural killer cells and inflammation produced antitumour effects for human oesophageal carcinoma cells. Scand J Immunol 2008; 68: 22-29.

41. Morishima N, Owaki T, Asakawa M, Kamiya S, Mizuguchi J, Yoshimoto T. Augmentation of effector CD8+ T cell generation with enhanced granzyme B expression by IL-27. J Immuno 2005; 175: 1686-1693.

42. Schneider R, Yaneva T, Beauseigle D, El-Khoury L, Arbour N. IL-27 increases the proliferation and effector functions of human naive $\mathrm{CD} 8+\mathrm{T}$ lymphocytes and promotes their development into Tc1 cells. Eur J Immunol 2011; 41: 47-59.

43. Yoshimoto T, Morishima N, Mizoguchi I, Shimizu M, Nagai H, Oniki S et al. Antiproliferative activity of IL-27 on melanoma. J Immunol 2008; 180: 6527-6535.

44. Shimizu M, Shimamura M, Owaki T, Asakawa M, Fujita K, Kudo M et al. Antiangiogenic and antitumor activities of IL-27. J Immunol 2006; 176: 7317-7324.

45. Pearl JE, Khader SA, Solache A, Gilmartin L, Ghilardi N, deSauvage F et al. IL-27 signaling compromises control of bacterial growth in Mycobacteria-infected mice. J Immunol 2004; 173: $7490-7496$

\section{Supplementary Information accompanies this paper on Cell Death and Differentiation website (http://www.nature.com/cdd)}

